\title{
ON ONE RELATOR GROUPS AND HNN EXTENSIONS
}

\author{
Dedicated to the memory of Hanna Neumann \\ JAMES MCCOOL ${ }^{1}$ and PAUL E. SCHUPP ${ }^{2}$
}

(Received 8 August 1972)

Communicated by G. E. Wall

In his work [5] on subgroups of one relator groups, Moldavanski observed that if $G$ is a one relator group whose defining relator $R$ is cyclically reduced and has exponent sum zero on some generator occurring in it, then $G$ is an $H N N$ extension of a one relator group $H$ whose defining relator is shorter than $R$. This observation, together with Britton's Lemma, can be used to give rather easy proofs of the basic results on one relator groups. To exposit this point of view, we give here a proof of the Freiheitssatz, the solvability of the word problem for one relator groups, and the theorem classifying elements of finite order in one relator groups. In particular, the solution obtained for the word problem is often easy to apply. We also give a proof of the "Spelling Theorem" of Newman [6].

We first recall the facts we need about the $H N N$ construction. Let $H=\langle S ; D\rangle$ be a group, and let $X$ and $Y$ be isomorphic subgroups of $H$ with $\theta: X \rightarrow Y$ an isomorphism. The group

$$
G=\left\langle S, t ; D, t x t^{-1}=\theta(x), x \in X\right\rangle,
$$

where $t$ is a letter not in $S$, is called an HNN group (or an HNN extension) with base $H$ and stable letter $t$. The $H N N$ construction was introduced by Higman, Neumann, and Neumann [2], who proved that $H$ is embedded in $G$ by the map $s \rightarrow s(s \in S)$. The "normal form theorem" for $H N N$ groups was discovered by Britton (Lemma 4 of [1]) and is known as Britton's Lemma (for a straightforward proof see [4]). If $w$ is a word on $\{S, t\}$, then $w$ is said to be $t$-free if $t$ does not occur in $w$. Britton's Lemma says that if $w$ is a word of $G$ in which $t$ occurs, and $w=1$ in $G$, then $w$ contains a subword of the form $t x t^{-1}$ where $x$ is $t$-free and $x \in X$, or $w$ contains a subword of the form $t^{-1} y t$ where $y$ is $t$-free and

1 Research supported by the National Research Council of Canada.

2 Research supported by National Science Foundation. 
$y \in Y$. A word on $\{S, t\}$ is said to be $t$-reduced if it does not contain a subword of either of the above forms. It follows from Britton's Lemma that if $w=u$ in $G$ where $w$ is $t$-reduced and $u$ is $t$-free, then $w$ is $t$-free. A word $w$ on $\{S, t\}$ is said to be cyclically $t$-reduced if all cyclic permutations of $w$ are $t$-reduced. If $w$ is cyclically $t$-reduced and $t$ occurs in $w$ then all powers $w^{n}(n \geqq 1)$ of $w$ are $t$-reduced words involving $t$. It follows that $w$ has infinite order in $G$. Since any element of $G$ is conjugate to a cyclically $t$-reduced word, any element of finite order of $G$ is conjugate to an element of finite order of $H$.

The generalized word problem for a subgroup $K$ of a recursively presented group $H=\langle S ; D\rangle$ is solvable if there exists an algorithm which, given any word $w$ on $S$, determines whether or not $w \in K$. We shall simply say that $K$ is solvable in $H$ if the generalized word problem for $K$ in $H$ is solvable. Note that if $H$ has solvable word problem, if $X$ and $Y$ are solvable subgroups of $H$, and if the isomorphism $\theta: X \rightarrow Y$ is recursive, then the word problem for $G=\left\langle S, t ; D, t x t^{-1}\right.$ $=\theta(x), x \in X\rangle$ is solvable. For, given any word $w$ on $\{S, t\}$, by repeating a finite number of times the operation of $t$-reduction, i.e., by replacing a subword of the form $t x t^{-1}$, where $x$ is $t$-free and $x \in X$, by $\theta(x)$, or replacing a subword $t^{-1} y t$, where $y$ is $t$-free and $y \in Y$, by $\theta^{-1}(y)$, one arrives at a $t$-reduced word $w^{*}$ with $w^{*}=w$ in $G$. (The operation of $t$-reduction is effective if the assumptions of the previous sentence hold). Now $w^{*}$ either contains $t$, in which case $w^{*} \neq 1$ in $G$, or $w^{*}$ is $t$-free and the question of whether or not $w^{*}=1$ is reduced to the word problem for $H$.

THEOREM 1. (The Freiheitssatz). Let $G=\left\langle t, b_{0}, c_{0}, \cdots ; R\right\rangle$ where $R$ is cyclically reduced. If $L$ is a subset of $\left\{t, b_{0}, c_{0}, \cdots\right\}$ which omits a generator occurring in $R$ then the subgroup $K$ generated by $L$ is freely generated by $L$.

Proof. It suffices to consider sets $L$ containing all the generators of $G$ except one. The proof is by induction on the length of $R$. If $R$ involves only one generator the theorem holds. We may thus assume that $G$ has at least two generators, $t$ and $b_{0}$, occurring in $R$. There are two cases.

Case 1. Suppose that the exponent sum, $\sigma_{t}(R)$, of some generator $t$ occurring in $R$ is zero. We exhibit $G$ as an $H N N$ extension of a one relator group $H$ whose defining relator $P$ has shorter length than $R$. We can assume, replacing $R$ by a suitable cyclic permutation if necessary, that $R$ begins with $b_{0}^{ \pm 1}$. For $i$ an integer, we put $b_{i}=t^{i} b_{0} t^{-i}, c_{i}=t^{i} c_{0} t^{-i}$, etc. As an element of the free group $F=\left\langle t, b_{0}, c_{0}, \cdots\right\rangle, R$ belongs to the normal subgroup of $F$ generated by $b_{0}, c_{0}, \cdots$, and so, $R$ can be rewritten as a cyclically reduced word $P$ on the $b_{i}, c_{i}, \cdots$, where the length of $P$ is less than that of $R$. To rewrite $R$, simply replace each occurrence of a generator other than $t$ by the generator subscripted by $i$, where $i$ is the sum of the exponents on $t$ 's preceding the given occurrence of the generator. (For example, if $R$ is $b_{0}^{2} t^{-1} c_{0}^{2} b_{0}^{2} t c_{0}^{2}$, then $P$ is $b_{0}^{2} c_{-1}^{2} b_{-1}^{2} c_{0}^{2}$.) 
Let $\tau$ and $s$ be respectively the minimum and maximum subscripts on $b$ actually occurring in $P$. (Note that $b_{0}$ occurs in $P$ since we are assuming that $R$ starts with $\left.b_{0}^{ \pm 1}\right)$. We claim that $G$ has presentation

$$
\begin{array}{r}
\left\langle t, b_{\tau}, \cdots, b_{s}, c_{i}, d_{i}, \cdots,(i \in Z) ; P=1, t b_{j} t^{-1}=b_{j+1}, \quad(j=\tau, \cdots, s-1),\right. \\
\left.t c_{i} t^{-1}=c_{i+1}, \cdots,(i \in Z)\right\rangle
\end{array}
$$

To see this, let $G^{*}$ be the group given by the new presentation. The map $\phi: G \rightarrow G^{*}$ defined by $t \rightarrow t, b_{0} \rightarrow b_{0}, c_{0} \rightarrow c_{0}, \cdots$ is a homomorphism since $\phi(R)=P$. On the other hand, the map $\eta: G^{*} \rightarrow G$ defined by $t \rightarrow t, p_{i} \rightarrow t^{i} b_{0} t^{-i}, c_{i} \rightarrow t^{i} c_{0} t^{-i}, \cdots$ is a homomorphism since all the relators of $G^{*}$ are sent to 1 . Since $\eta \phi$ and $\phi \eta$ are the identity maps on $G$ and $G^{*}$ respectively, $\phi$ is an isomorphism. Continuing our example, if

$$
G=\left\langle t, b_{0}, c_{0} ; b_{0}^{2} t^{-1} c_{0}^{2} b_{0}^{2} t c_{0}^{2}\right\rangle,
$$

the new presentation of $G$ is

$$
\left\langle t, b_{-1}, b_{0}, c_{i}(i \in Z) ; b_{0}^{2} c_{-1}^{2} b_{-1}^{2} c_{0}^{2}, t b_{-1} t^{-1}=b_{0}, t c_{i} t^{-1}=c_{i+1}(i \in Z)\right\rangle .
$$

Now put $H=\left\langle b_{\tau}, \cdots, b_{s}, c_{i}, d_{i}, \cdots,(i \in Z) ; P=1\right\rangle$. It follows from the induction hypothesis that the subgroups $X$ and $Y$ of $H$ generated respectively by $\left\{b_{\tau}, \cdots, b_{s-1}, c_{i}, d_{i} \cdots,(i \in \boldsymbol{Z})\right\}$ and $\left\{b_{\tau+1}, \cdots, b_{s}, c_{i}, d_{i}, \cdots,(i \in \boldsymbol{Z})\right\}$ are freely generated by the indicated generators. In particular, the map

$$
b_{j} \rightarrow b_{j+1}, c_{i} \rightarrow c_{i+1}, \cdots,(\tau \leqq j<s, i \in Z)
$$

extends to an isomorphism $\theta: X \rightarrow Y$. Thus $G$ is exhibited as $H N N$ group with base $H$.

First suppose that our subset $L$ of the original generators for $G$ is $L=\left\{b_{0}\right.$, $\left.c_{0}, \cdots\right\}$. Note that at least one generator of $H$ with a non-zero subscript occurs in $P$ (for otherwise $t$ could not occur in $R$ with zero exponent sum). By the induction hypothesis $L$ freely generates a free subgroup of $H$, and thus of $G$.

Suppose that $L=\left\{t, c_{0}, \cdots\right\}$. (The omitted generator is $b_{0}$ ). Let $w$ be a nontrivial freely reduced word on $\left\{t, c_{0}, \cdots\right\}$. If $\sigma_{t}(w) \neq 0$, then $w \neq 1$ in $G$. (Any word equal to 1 in $G$ must be freely equal to a product of conjugates of $R^{ \pm 1}$ ). If $\sigma_{t}(w)=0$, rewrite $w$ as a word on the set $J=\left\{c_{i}, \cdots\right\}$, following the same procedure as in rewriting $R$. This yields a freely reduced non-trivial word $w^{*}$. By the induction hypothesis $J$ freely generates a free subgroup of $H$. Hence, $w^{*} \neq 1$. Since $w^{*}=w$ in $G, w \neq 1$ in $G$.

Case 2. Up to a relabelling of generators, Case 1 covers all situations except that where all the generators occurring in $R$ have non-zero exponent sum. Suppose $L=\left\{b_{0}, c_{0}, \cdots\right\}$. Let $\sigma_{t}(R)=\alpha$, and let $\sigma_{b_{0}}(R)=\beta$. The map $\Psi$ defined by $t \rightarrow y x^{-\beta}, b_{0} \rightarrow x^{\alpha}, c_{0} \rightarrow c_{0}, \cdots$ is a homomorphism of $G$ into the group 


$$
C=\left\langle y, x, c_{0}, \cdots ; R\left(y x^{-\beta}, x^{\alpha}, c_{0}, \cdots\right)\right\rangle \text {. }
$$

Let $R_{1}$ be the result of cyclically reducing $R\left(y x^{-\beta}, x^{\alpha}, c_{0}, \cdots\right)$. Then $\sigma_{x}\left(R_{1}\right)=0$ and $y$ occurs in $R_{1}$. We can rewrite $C$ as an $H N N$ group using $x$ as a stable letter. If $P$ is the rewritten form of $R\left(y x^{-\beta}, x^{\alpha}, c_{0}, \cdots\right)$ then the length of $P$ will be less than the length of $R$ since all $x$ symbols will be eliminated. It follows that the subgroup of $C$ generated by $\left\{x, c_{0}, \cdots\right\}$ is freely generated by this set and thus the subgroup generated by $\left\{x^{\alpha}, c_{0}, \cdots\right\}$ is freely generated by these generators. Since $\Psi$ sends $b_{0} \rightarrow x^{\alpha}, c_{0} \rightarrow c_{0}, \cdots, L$ freely generates a free subgroup of $G$. This completes the proof of the theorem.

As G. Sacerdote pointed out to us, Britton's Lemma is not used in the proof of the Freiheitssatz. Only the fact that the base group is embedded in an $H N N$ extension is needed.

By the Freiheitssatz, the element $b_{0}$ has infinite order in $G$. Thus the group $C^{\prime}=\left\langle t, b_{0}, c_{0} \cdots, x ; R, b_{0}=x^{\alpha}\right\rangle$ is the free product of $G$ and the infinite cyclic group $\langle x\rangle$, amalgamating $b_{0}=x^{\alpha}$. $C$ is obtained from $C^{\prime}$ by the Tietze transformations of adding a new generator $y$ and relation $t=y x^{-\beta}$, and then deleting the generators $b_{0}$ and $t$ and replacing them in $R$ by $x^{\alpha}$ and $y x^{-\beta}$. Thus the map $\Psi: G \rightarrow C$ is an embedding.

We turn to the theorem characterizing elements of finite order in one relator groups.

THEOREM 2. Let $G=\left\langle t, b_{0}, c_{0}, \cdots ; R\right\rangle$ where $R$ is cyclically reduced. The group $G$ is torsion free if $R$ is not a proper power. If $R=U^{n}, n>1$, where $U$ itself is not a proper power, then $U$ has order $n$ in $G$ and all elements of finite order of $G$ are conjugates of powers of $U$.

Proof. This theorem is almost immediate from the remarks characterizing elements of finite order in $H N N$ groups. The proof is, of course, by induction on the length of $R$. If $R$ involves only one generator, the theorem holds. We may thus assume that $R$ involves at least two generators, say $t$ and $b_{0}$. If $R$ has exponent sum zero on some generator, say $t$, then, as before, we consider $G$ as an $H N N$ group with base

$$
H=\left\langle b_{\tau}, \cdots, b_{s}, c_{i}, \cdots(i \in Z) ; P=1\right\rangle
$$

where $P$ is the rewritten form of $R$. Note that $P$ is an $n$-th power if and only if $R$ is an $n$-th power. By the induction hypothesis, $H$, and thus $G$, is torsion free unless $R$ is a proper power. If $R=U^{n}, n>1$, (where $U$ is not a proper power), then $P=V^{n}$ where $V$ is the rewritten form of $U$. The induction hypothesis guarantees that the only elements of finite order in $H$, and thus $G$, are conjugates of powers of $V$ and that $V$ has order $n$. Since $V=U$ in $G$ we are done.

In the case where no generator in $R$ has exponent sum zero, we must consider the group 


$$
C=\left\langle y, x, c_{0}, \cdots ; R\left(y x^{-\beta}, x^{\alpha}, c_{0}, \cdots\right)\right\rangle .
$$

If $R=U^{n}$ then the result of cyclically reducing $R\left(y x^{-\beta}, x^{\alpha}, c_{0}, \cdots\right\}$ is of the form $U_{1}^{n}$. Since the map $\Psi$ (defined earlier) from $G$ to $C$ is an embedding, and $\Psi(U)$ is conjugate to $U_{1}$, the result follows as in case 1 .

THEOREM 3. Let $G=\left\langle t, b_{0}, c_{0}, \cdots ; R\right\rangle$ be a countably generated one relator group where $R$ is cyclically reduced. If $K$ is the subgroup generated by a recursive subset $L$ of the given generators of $G$ then the generalized word problem with respect to $K$ in $G$ is solvable.

Proof. Since $G$ is the free product of the one relator group generated by the generators which occur in $R$ and the free group on the remaining generators, the result is clear if $L$ contains all the generators occurring in $R$. We need only show that if $L$ is a maximal subset which omits a generator occurring in $R$, then the subgroup $K$ generated by $L$ is solvable in $G$. For, by the Freiheissatz, $K$ is freely generated by $L$. Given a recursive subset $J$ of $L$, the subgroup generated by $J$ is clearly solvable in $K$ : and thus also in $G$ provided that $K$ is solvable in $G$.

The proof is by induction on the length of $R$. If $R$ involves only one generator the theorem holds since $G$ is then the free product of a free group and a cyclic group. First suppose that a generator occurring in $R$, say $t$, has $\sigma_{t}(R)=0$. As before, consider $G$ as an $H N N$ extension with base $H$.

Suppose that $K$ is the subgroup generated by the subset $L=\left\{b_{0}, c_{0}, \cdots\right\}$ of the original generators. By the induction hypothesis, the subgroups $X$ and $Y$ used to exhibit $G$ as an $H N N$ extension of $H$ are solvable in $H$. Performing $t$-reductions is therefore an effective process. Thus, given any word $w$ on $\left\{t, b_{0}, c_{0}, \cdots\right\}$, we can effectively calculate a $t$-reduced word $w^{*}$ with $w^{*}=w$ in $G$. By Britton's Lemma, if $w^{*}$ is equal in $G$ to a word on $\left\{b_{0}, c_{0}, \cdots\right\}$ then $w^{*}$ must be $t$-free. Now $K$ is solvable in $H$ by the induction hypothesis. Since $w^{*} \in K$ if and only if $w \in K, K$ is solvable in $G$.

Suppose that $K$ is the subgroup generated by $L=\left\{t, c_{0}, \cdots\right\}$. Let $w$ be a word on $\left\{t, b_{0}, c_{0} \cdots\right\}$ and let $\alpha=\sigma_{t}(w)$. Then $w \in K$ if and only if $w t^{-\alpha} \in K$. Suppose that $w t^{-\alpha}$ is equal to a word $v$ on $L=\left\{t, c_{0}, \cdots\right\}$. By Britton's Lemma, we must have $\sigma_{t}(v)=0$ since $w t^{-\alpha}$ has exponent sum zero in $t$. Since $L$ does not contain $b_{0}, v$ may be rewritten (as in rewriting $R$ ) as a $t$-free word $v^{*}$ on $J=\left\{c_{i}, \cdots(i \in \boldsymbol{Z}).\right\}$

An equation of the form $w t^{-\alpha}=v^{*}$ is possible only if successive $t$-reductions lead from $w t^{-\alpha}$ to a $t$-free word $w^{*}$. The subgroup $N$ generated by $J$ is solvable in $H$ by the induction hypothesis. Since $w \in K$ if and only if $w^{*} \in N, K$ is solvable in $G$.

Finally suppose that all generators occurring in $R$ have non-zero exponent sum and that $K$ is the subgroup generated by $L=\left\{b_{0}, c_{0}, \cdots\right\}$. We again consider the group $C$. Since the map $\Psi: G \rightarrow C$ is an embedding, a word $w\left(t, b_{0}, c_{0}, \cdots\right) \in K$ 
if and only if $w\left(y x^{-\beta}, x^{-\alpha}, c_{0}, \cdots\right) \in \Psi(K)$. As in the previous case, the subgroup $M$ generated by $\left\{x, c_{0}, \cdots\right\}$ is solvable in $C$. Since $M$ is freely generated by these generators, $\Psi(K)$, which is the subgroup generated by $\left\{x^{\alpha}, c_{0}, \cdots\right\}$, is solvable in $M$ and thus $C$. Thus $K$ is solvable in $G$. This concludes the proof of the theorem.

In the case of a one relator group with torsion, the "Spelling Theorem" of Newman [6] gives a much sharper solution to the word problem. We conclude the paper with a proof of this result.

THEOREM 4. Let $G=\left\langle t, b_{0}, c_{0}, \cdots ; R^{n}=1\right\rangle$ where $R$ is cyclically reduced and $n>1$. Suppose $w=v$ in $G$ where $w$ is a freely reduced word on $\left\{t, b_{0}, c_{0}, \cdots\right\}$ and $v$ omits a generator occurring in $w$. Then $w$ contains a subword $Q$ such that $Q$ is also a subword of $R^{ \pm n}$ and the length of $Q$ is greater than $(n-1) / n$ times the length of $R^{n}$.

Proof. First observe that since $G$ is the free product of the group on those generators actually occurring in $R$ and the free group $F$ on the remaining generators, an equation $w=v$ where $w$ is freely reduced and $v$ omits a generator occurring in $w$ cannot hold in $G$ if the omitted generator does not occur in $R$. We thus need consider only equations in which the omitted generator occurs in the defining relation $R$. The proof, of course, is by induction on the length of $R$. If $R$ involves only one generator the result holds, so we may assume that $R$ involves at least two generators.

Case 1. We first consider the case where some generator $t$ occurring in $R$ has $\sigma_{t}(R)=0$. As before, we consider $G$ as an $H N N$ group with base

$$
H=\left\langle b_{\tau}, \cdots, b_{s}, c_{i}, \cdots(i \in Z) ; P^{n}=1\right\rangle .
$$

The subgroups $X$ and $Y$ which are conjugated by $t$ are freely generated by $\left\{b_{\imath}, \cdots, b_{s-1}, c_{i}, \cdots(i \in \boldsymbol{Z})\right\}$ and $\left\{b_{\imath+1}, \cdots, b_{s}, c_{i}, \cdots(i \in \boldsymbol{Z})\right\}$ respectively.

Suppose that $w$ is a freely reduced word on $t, b_{0}, c_{0}, \cdots$, and that $w\left(t, b_{0}, \cdots\right)$ $=v\left(b_{0}, \cdots\right)$ where $t$ occurs in $w$ but not in $v$. The equation $w\left(t, b_{0}, \cdots\right)=v\left(b_{0}, \cdots\right)$ implies that $w$ can be reduced to a $t$-free word $w^{*}$ by $t$-reductions. By a $t$-reduction which is simply a "shifting of subscripts" we mean replacing a subword $t^{\varepsilon} u\left(b_{i}, c_{i}, \cdots\right) t^{-\varepsilon}, \varepsilon= \pm 1$, by $u\left(b_{i+\varepsilon}, c_{i+\varepsilon}, \cdots\right)$ where all the generators occurring in $u$ occur explicitly among the given generators of $X$ (if $\varepsilon=1$ ) or $Y$ (if $\varepsilon=-1$ ). The point of considering such reductions is that if $w^{\prime}$ is obtained from $w$ by $t$-reductions which simply shift subscripts, then $w$ can be obtained from $w^{\prime}$ by replacing each $b_{1}, c_{i}, \cdots$ in $w^{\prime}$ by $t^{i} b_{0} t^{-i}, t^{i} c_{0} t^{-i}, \cdots$, and then freely reducing, and the only letters cancelled in the free reductions will be $t$-symbols.

Starting with the original word $w$ perform, wherever possible, $t$-reductions which simply shift subscripts. Suppose we reach a word $w^{\prime}$ to which no such 
reductions can be applied but $w^{\prime}$ is not $t$-reduced. Then $w^{\prime}$ contains a subword $t^{\varepsilon} u t^{-\varepsilon}$ where $u$ is $t$-free and $u$ itself is not a word on the given generators for $X$ or $Y$ (depending on $\varepsilon$ ) but $u$ is equal to a word $z$ on the given generators. Thus $z$ omits a generator of $H$ occurring in $u$, and $u=z$ in $H$. By the induction hypothesis, $u$ contains a subword $Q$ of $P^{ \pm n}$ of the required length. Since we have only shifted subscripts to obtain $w^{\prime}$, we can recover $w$ from $w^{\prime}$ by replacing each $b_{i}, \cdots$, by $t^{i} b_{0} t^{-i}$ and freely reducing, cancelling only $t$-symbols. Note that the part $S$ of $u$ which is recovered from $Q$ will contain a subword of $R^{ \pm n}$ of the desired length even if we disregard any occurrences of $t^{ \pm 1}$ at the beginning or end of $S$.

If $w$ can be reduced to a $t$-free word $w^{*}$ by shifting subscripts, then $w^{*}$ must contain a generator with a non-zero subscript and $w^{*}=v$ in $H$. By the induction hypothesis, $w^{*}$ must contain a subword $Q$ of $P^{ \pm n}$ of the desired length. As before, this implies that $w$ contains a suitable subword $S$ of $R^{ \pm n}$ and, moreover, $S$ can be chosen so that it does not begin or end with $t^{ \pm 1}$. (We shall need later the fact that the subword $S$ can be chosen not to begin or end with the stable letter $t$ ).

Next suppose that an equation $w\left(t, b_{0}, c_{0}, \cdots\right)=v\left(t, c_{0}, \cdots\right)$ holds in $G$, where $b_{0}$ occurs in $w$ but not in $v$. By Britton's Lemma, we have $\sigma_{t}(w)=\sigma_{t}(v)=\alpha$ say. Hence $w t^{-\alpha}=v t^{-\alpha}$. Since $v t^{-\alpha}$ has exponent sum zero on $t$ and does not contain any $b_{i}$-symbols, $v t^{-\alpha}$ can be rewritten as a $t$-free word $v^{*}$ by shifting subscripts. The equation $w t^{-\alpha}=v^{*}$ holds in $G$. As in the previous argument, if $w t^{-\alpha}$ cannot be reduced to a $t$-free word by shifting subcripts we conclude that $w t^{-\alpha}$ must contain a subword $S$ of $R^{ \pm n}$ of the desired length where $S$ does not begin or end with $t^{ \pm 1}$. The latter condition ensures that $S$ is a subword of $w$. If $w t^{-\alpha}$ can be reduced to a $t$-free word $w^{*}$ by shifting subscripts, then some $b_{i}$ occurs in $w^{*}$. Since $w^{*}=v^{*}$ in $H$, the induction hypothesis says that $w^{*}$ contains a subword $Q$ of $P^{ \pm n}$ of the desired length. As in the first part of this paragraph, $w$ must then contain a subword $S$ of $R^{ \pm n}$ of the desired length where $S$ does not begin or end with $t^{ \pm 1}$.

Case 2. Finally, suppose that all generators occurring in $R$ have non-zero exponent sum. Suppose that an equation $w\left(t, b_{0}, \cdots\right)=v\left(b_{0}, \cdots\right)$ holds in $G$ where $t$ occurs in $w$ but not $v$. Let $\alpha=\sigma_{t}(R)$ and let $\beta=\sigma_{b_{0}}(R)$. The map $\Psi$ defined by $t \rightarrow y x^{-\beta}, b_{0} \rightarrow x^{\alpha}, c_{0} \rightarrow c_{0}, \cdots$ is an embedding of $G$ into the group

$$
C=\left\langle y, x, c_{0}, \cdots ; R^{n}\left(y x^{-\beta}, x^{\alpha}, c_{0}, \cdots\right)\right\rangle
$$

Let $w^{\prime}$ be the result of freely reducing $w\left(y x^{-\beta}, x^{\alpha}, c_{0}, \cdots\right)$ and let $v^{\prime}$ be $v\left(x^{\alpha}, c_{0}, \cdots\right)$. Now $w^{\prime}$ contains an occurrence of $y$ and $v^{\prime}$ does not. As in case $1, w^{\prime}$ contains a subword $Q^{\prime}$ of $R^{ \pm n}\left(y x^{-\beta}, x^{\alpha}, c_{0}, \cdots\right)$ of the desired length, and $Q^{\prime}$ does not begin or end with $x^{ \pm 1}$. This implies that $w$ contains a suitable subword of $R^{ \pm n}$. This concludes the proof of the theorem. 


\section{Bibliography}

[1] J. L. Britton, 'The word problem', Annals of Math., 77 (1963), 16-32.

[2] Graham Higman, B. H. Neumann, and Hanna Neumann, 'Embedding theorems for groups', J. London Math. Soc. 24J (1949), 247-254.

[3] W. Magnus, A. Karrass, and D. Solitar, Combinatorial group theory, (Interscience, New York, 1966.)

[4] C. F. Miller III, 'On Britton's theorem A', Proc. Amer, Math. Soc. 19 (1968), 1151-1154.

[5] D. I. Moldavanskii, 'Certain subgroups of groups with one defining relator', Siberian Math. J. 8 (1967), 1370-1384.

[6] B. B. Newman, 'Some results on one-relator groups', Bull. Amer. Math. Soc. 74 (1968), 568-571.

[7] B. B. Newman, Some aspects of one-relator groups, (Ph.D. Thesis, University of Queensland, 1968).

Department of Mathematics

University of Toronto

Toronto 181

Canada

Department of Mathematics

University of Illinois

Urbana, Illinois 61801

U. S. A. 